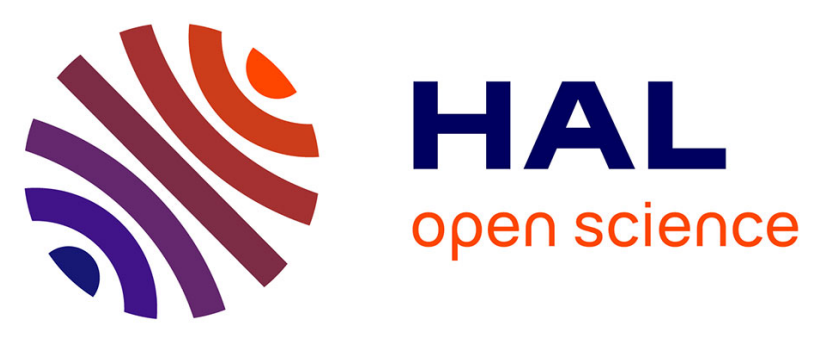

\title{
Rôle des propriétés physiques du lit de semences sur l'imbibition et la germination. III. Valeur prévisionnelle d'un modèle d'imbibition au champ et caractérisation des lits de semences
}

\author{
Jean Boiffin, Laurent Bruckler, Christine Aubry
}

\section{To cite this version:}

Jean Boiffin, Laurent Bruckler, Christine Aubry. Rôle des propriétés physiques du lit de semences sur l'imbibition et la germination. III. Valeur prévisionnelle d'un modèle d'imbibition au champ et caractérisation des lits de semences. Agronomie, 1983, 3 (4), pp.291-302. hal-00884512

\section{HAL Id: hal-00884512 \\ https://hal.science/hal-00884512}

Submitted on 1 Jan 1983

HAL is a multi-disciplinary open access archive for the deposit and dissemination of scientific research documents, whether they are published or not. The documents may come from teaching and research institutions in France or abroad, or from public or private research centers.
L'archive ouverte pluridisciplinaire HAL, est destinée au dépôt et à la diffusion de documents scientifiques de niveau recherche, publiés ou non, émanant des établissements d'enseignement et de recherche français ou étrangers, des laboratoires publics ou privés. 


\title{
Rôle des propriétés physiques du lit de semences sur l'imbibition et la germination. III. Valeur prévisionnelle d'un modèle d'imbibition au champ et caractérisation des lits de semences
}

Jean BOIFFIN, Laurent BRUCKLER(*) \& Christine AUBRY

I.N.R.A., Laboratoire de Recherches de la Chaire d'Agronomie de l'I.N.A.P.G., 16, rue Claude-Bernard, F 75231 Paris Cedex 05.

(*) I.N.R.A., Station de Science du Sol, Centre de Recherches d'Avignon, B.P. 91, F 84140 Montfavet.

RÉSUMÉ

\begin{abstract}
On propose de tester au champ la valeur prévisionnelle d'un modèle d'imbibition des semences de mais. Pour cela, on réalise une série de traitements permettant d'analyser le rôle du potentiel de l'eau et de l'état structural au contact de la semence sur l'imbibition et la germination.

L'accord entre les prévisions et les observations portant sur des critères essentiels du point de vue agronomique (possibilité de germination, durée moyenne d'imbibition) est très satisfaisant. Le modèle prévisionnel testé est adapté à des conditions non isothermes : dans ce cas, le faisceau prévisionnel des cinétiques d'imbibition correspondant à la gamme d'états structuraux au contact des semences est compatible avec la variabilité des réponses des semences observées in situ. Une amélioration de la prévision amène d'une part, à tester le modèle sur une gamme plus large d'états structuraux, d'autre part, à mieux prendre en compte le rôle des variations de température sur les propriétés physiques de la semence.
\end{abstract}

Mots-clés additionnels : Maüs, Modélisation, Humidité du sol, Potentiel matriciel, Structure. and imbibition model and characterization of seed-bed.

\begin{abstract}
A comparison between estimated values for imbibition of maize seeds and field experimental data is proposed. Experiments are introduced in view of analyse the roles of the soil-water potential and seed-soil contact area on the imbibition and germination.

Comparison between estimated values and field experimental data is satisfactory, according to the most important field tests (possibility of germination, mean duration of imbibition). The model is adapted to non-isothermal conditions : In this case, an agreement has been reached between the range of estimated values and the variability of experimental data, according to various seed-soil contact area. The improvement of the model requires to test this one for a larger range of seeds-beds structure, to take better into account the role of temperature on physical properties of seeds.
\end{abstract}

Additional key-words : Maize, Modelisation, Soil moisture, Maxtrix potential, Structure.

\section{INTRODUCTION}

Un modèle d'imbibition des semences de maïs permettant de prévoir, dans diverses conditions d'état structural et d'humidité du sol, l'imbibition des semences et leur possibilité de germination a été présenté précédemment (BRUCKLER, 1983 I, II).

Dans le présent travail, on se propose d'étudier les possibilités d'extrapolation au champ des conclusions acquises lors de la phase d'élaborátion théorique et de contrôle expérimental du modèle au laboratoire. Par extrapolation au champ, il faut entendre que l'on essaiera de tester la robustesse du modèle en contrôlant sa valeur prévisionnelle, dans des situations où toutes les conditions réalisées lors de son établissement ne sont plus simultanément vérifiées. Le contexte expérimental se rapproche ainsi de celui du champ cultivé et les principales différences par rapport aux conditions de laboratoire se ramènent à :

- l'absence d'une condition stricte d'isothermie à $20^{\circ} \mathrm{C}$, 
- une variabilité de l'état structural des lits de semences qui ne porte plus seulenıent sur la porosité structurale mais aussi sur la distribution dimensionnelle des agrégats,

- une modification du lot de semences utilisées et du substrat terreux.

La variété et le type de tri des semences restent identiques à ceux utilisés en laboratoire et la classe texturale choisie reste proche de la précédente.

Un prolongement attendu de ce travail est une amélioration des méthodes d'observation et de diagnostic concernant les semis.

\section{RAPPEL DU MODĖLE ET CONSÉQUENCES}

Le modèle proposé s’écrit de la façon suivante :

$$
\begin{gathered}
\left(\frac{\Delta \mathbf{H}_{s}}{\Delta \mathrm{t}}\right)_{t}=-\alpha_{1} \cdot \mathrm{K}^{\prime}\left(\mathrm{H}_{\mathrm{S}}\right) \cdot \mathrm{S}_{1} \cdot\left(\left|\psi_{\mathrm{s}}\right|-\left|\psi_{\mathrm{e}}\right|\right)-\alpha_{\mathrm{v}} \cdot \mathrm{D}^{\prime}\left(\mathrm{H}_{\mathrm{s}}\right) \cdot \mathrm{S}_{\mathrm{v}} \cdot\left(\mathrm{p}_{\mathrm{s}}-\mathrm{p}_{\mathrm{e}}\right) \\
\left(\frac{\Delta \mathrm{Hs}}{\Delta \mathrm{t}}\right)_{\mathrm{t}}: \begin{array}{l}
\text { variation d'humidité massique de la semence } \\
\text { pendant } \Delta \mathrm{t} .
\end{array}
\end{gathered}
$$

$\alpha_{1}$

$$
\begin{aligned}
& \text { : constante relative aux transferts en phase } \\
& \text { liquide. }
\end{aligned}
$$

$\alpha_{\mathrm{v}} \quad$ : constante relative aux transferts en phase gazeuse.

$K^{\prime}\left(H_{s}\right)$ : fonction d'ajustement relative aux transferts en phase liquide, caractéristique de la semence.

D' $\left(\mathrm{H}_{\mathrm{s}}\right)$ : fonction d'ajustement relative aux transferts en phase gazeuse, caractéristique de la semence.

$S_{1} \quad$ : surface d'imbibition en phase liquide.

$\mathrm{S}_{\mathrm{v}} \quad$ : surface d'imbibition en phase gazeuze.

$S_{1}+S_{v}=S_{t}$ : surface totale de la semence.

$\left(\left|\psi_{\mathrm{s}}\right|-\left|\psi_{\mathrm{e}}\right|\right),\left(\mathrm{p}_{\mathrm{s}}-\mathrm{p}_{\mathrm{e}}\right):$ différence de potentiel hydrique (phase liquide) ou de pression partielle de vapeur d'eau (phase gazeuse) entre la semence (indice « s ») et le milieu extérieur (indice « e »).

La connaissance de 2 types de variables impliquées dans le modèle est nécessaire pour simuler l'humectation des semences à partir de la relation (1) :

- d'une part, les variables relatives aux surfaces de la semence fonctionnelles en phase liquide $\left(S_{1}\right)$ ou en phase vapeur $\left(\mathrm{S}_{\mathrm{v}}\right)$,

- d'autre part, les variables relatives à la différence de potentiel $\left(\left|\psi_{\mathrm{s}}\right|-\left|\psi_{\mathrm{e}}\right|\right)$ ou de pression partielle de vapeur d'eau $\left(p_{s}-p_{e}\right)$ entre la semence et le milieu extérieur.

\section{A. Détermination des variables mises en jeu dans le modèle}

Les variables $\left(\left|\psi_{s}\right|-\left|\psi_{e}\right|\right)$ et $\left(p_{s}-p_{e}\right)$ peuvent être regroupées en variables relatives à la semence $\left(\left|\psi_{\mathrm{s}}\right|, \mathrm{p}_{\mathrm{s}}\right)$ ou au sol $\left(\left|\psi_{\mathrm{e}}\right|, \mathrm{p}_{\mathrm{e}}\right)$. La relation $\left|\psi_{\mathrm{s}}\right|=\mathrm{f}\left(\mathrm{H}_{\mathrm{s}}\right)$ relative au maïs et son analogue $\mathrm{p}_{\mathrm{s}}=\mathrm{f}\left(\mathrm{H}_{\mathrm{s}}\right)$ ont été déterminées expérimen- talement au cours d'un travail préliminaire (BRUCKLER, 1983 I) et nous admettrons que le changement de lot n'entraîne pas de modification notable de ces fonctions. La connaissance des variables $\left(\left|\psi_{\mathrm{e}}\right|, \mathrm{p}_{\mathrm{e}}\right)$ se ramènera à la mesure d'une teneur en eau $\mathrm{H}_{e}$ et à la détermination expérimentale de la relation $\left|\psi_{\mathrm{e}}\right|=\mathrm{f}\left(\mathrm{H}_{\mathrm{e}}\right)$, ou de son analogue $\mathrm{p}_{\mathrm{e}}=$ f $\left(\mathrm{H}_{\mathrm{e}}\right)$.

Le cas des variables $\left(S_{1}, S_{v}\right)$ est plus complexe puisqu'on ne connaît pas les valeurs individuelles $\left(S_{1}, S_{v}\right)$ d'une semence placée dans un sol. Cependant, dans le cas où les semences sont placées dans un milieu constitué d'agrégats homogènes et de petite taille $(2-3 \mathrm{~mm})$, à texture limoneuse, et lorsque l'alimentation en eau est non limitante, on obtient la relation linéaire suivante (BRUCKLER, 1983 II) :

$\mathrm{S}_{1} / \mathrm{S}_{\mathrm{t}}=0,94\left(1-\mathrm{n}_{\mathrm{s}}\right)-0,16 \quad \mathrm{n}=94 \quad \mathrm{r}^{2}=0,80$

$\mathrm{n}_{\mathrm{s}}$ : porosité structurale à proximité de la semence

$$
\mathrm{n}_{\mathrm{s}} \in[0 ; 45]
$$

Il semble ainsi possible d'estimer tout couple $\left(\mathrm{S}_{1}, \mathrm{~S}_{\mathrm{v}}\right)$ à partir de la description du système de porosité du lit de semences, lorsqu'on est assuré que l'imbibition correspond à un cas d'alimentation hydrique non limitante. L'utilisation de (2) pose cependant 2 problèmes :

a) la mesure de $n_{\mathrm{S}}$ à proximité de la semence suppose au préalable l'acquisition d'une technique de mesure à haute résolution spatiale.

b) la relation (2) est a priori contingente de la taille, du degré d'homogénéité, de la morphologie des agrégats entourant la semence.

L'utilisation de la relation (2) au cours de ce travail permettra précisément de tester la validité de l'estimation choisie pour une assez large gamme d'états structuraux.

\section{B. Cas types d'imbibition des semences}

La confrontation des données expérimentales aux données du modèle, telle qu'elle a été réalisée en sol limoneux, permet de proposer 3 cas typiques d'imbibition résumés dans le tableau 1.

La détermination de la valeur $\mathrm{x}$ du tableau 1 est fondamentale puisque le cas 1 correspond au strict domaine d'utilisation de la relation (2). Elle peut se faire selon 2 approches indépendantes :

\section{Ire approche : calcul préliminaire d'ordre de grandeur :}

On situe le domaine de teneurs en eau du sol pour lesquelles des gradients de potentiel hydrique relativement faibles autour de la semence, < 1 bar/cm (DASBERG, 1971), assurent vers celle-ci un débit maximal, égal à celui observé lors de l'immersion d'une semence dans l'eau. Les modalités de calcul et les résultats sont donnés par le tableau 2: ainsi estimée, la borne inférieure du domaine non limitant se situe approximativement vers la valeur $H_{e}=0,14\left(\left|\psi_{e}\right|\right.$ $\sim 2,7$ bar).

\section{$2^{e}$ approche : recherche expérimentale}

Par définition, dans le domaine non limitant, les cinétiques d'imbibition des semences sont identiques lorsque l'état structural est invariant. Les cinétiques d'imbibition réalisées dans un milieu agrégé homogène pour différentes 
TABLEAU 1

Cas types d'imbibition:

(l) la valeur $x$ limite les domaines limitant et non limitant et est fonction du type de sol.

(2) la valeur 16 bars est approchée; en fait, il s'agit d'une valeur de 12 à 16 bars selon les semences, qui sépare les domaines de germination possible ou impossible. (3) si le sol est très sec, $S_{l} / S_{t}=0$.

Typical cases of imbibition:

(I) the (x) value is a boundary between the ranges of limiting and not limiting soil-water contents.

This value depends of physical properties of soils.

(2) the 16 bars value is approached; the real value

is between 12 and 16 bars and this value is a boundary between soil-water contents where germination is possible or impossible. (3) if the soil is very dry, $S_{1} / S_{t}=0$.

\begin{tabular}{cccc}
\hline $\begin{array}{c}\text { Cas } \\
\text { expérimental }\end{array}$ & Cas 1 & Cas 2 & Cas 3 \\
\hline$\left|\psi_{\mathrm{e}}\right|$ & $.0 \mathrm{à} \mathrm{x}$ bars $(1)$ & $\mathrm{x}$ à 16 bars $(2)$ & $>16$ bars $(2)$ \\
\hline $\begin{array}{c}\text { Variables } \\
\text { conditionnant } \\
\text { l'imbibition }\end{array}$ & $\mathrm{S}_{\mathrm{l}} / \mathrm{S}_{\mathrm{t}}$ & $\left|\psi_{\mathrm{e}}\right|, \mathrm{p}_{\mathrm{e}}, \mathrm{S}_{\mathrm{l}} / \mathrm{S}_{\mathrm{t}}\left|\psi_{\mathrm{e}}\right|, \mathrm{p}_{\mathrm{e}}, \mathrm{S}_{\mathrm{l}} / \mathrm{S}_{\mathrm{t}}{ }^{\left({ }^{3}\right)}$
\end{tabular}

\section{Dénomination}

du domaine de

teneur en eau non limitant limitant limitant du sol

Germination possible possible impossible

valeurs de l'humidité $\mathbf{H}_{\mathrm{e}}$ (fig. 1) sont groupées lorsque $\mathbf{H}_{\mathrm{e}}$ $\geqslant 0,143\left(\left|\psi_{\mathrm{e}}\right| \leqslant 2,4\right.$ bars $)$. Elles se distinguent nettement

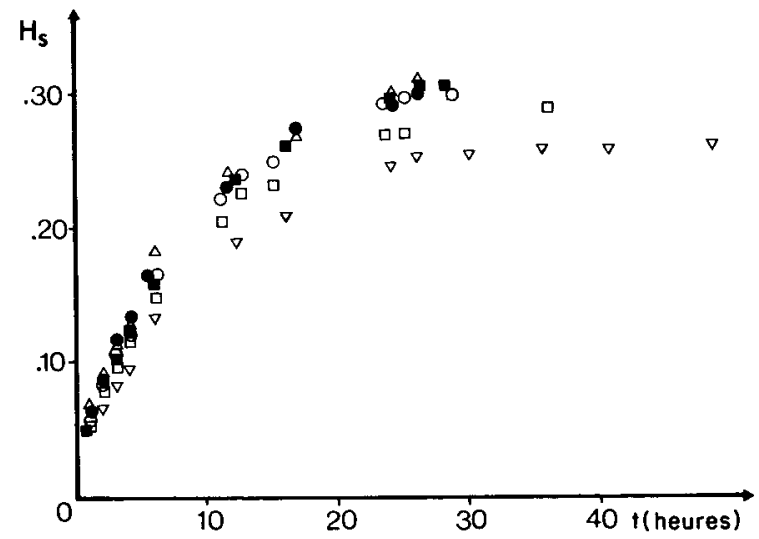

Figure 1

Imbibition des semences dans les massifs d'agrégats $(2-3 \mathrm{~mm})$ à différentes humidités initiales $\left(T=20^{\circ} \mathrm{C}\right)$ :

Imbibition of seeds in aggregates $(2-3 \mathrm{~mm})$ for different initial soil water-contents $\left(T=20^{\circ} \mathrm{C}\right)$ :

$\begin{array}{ccc} & \mathrm{H}_{\mathrm{e}} & \psi_{\mathrm{e}} \\ (\Delta): & 0,196 & 0,8 \mathrm{bar} \\ (\bullet): & 0,173 & 1,3 \mathrm{bar} \\ (\mathrm{O}): & 0,156 & 1,8 \text { bar } \\ (\square): & 0,143 & 2,4 \mathrm{bars} \\ (\square): & 0,110 & 6 \mathrm{bars} \\ (\nabla): & 0,083 & 16 \text { bars }\end{array}$

\section{TABLEAU 2}

Estimation des gradients hydriques autour de la semence nécessaires pour assurer un transfert maximal vers celle-ci:

(I) déterminée selon la méthode proposée par CAMPBELL (1974)

(2) différence de potentiel nécessaire pour assurer vers la semence un débit maximal $q(20 \mathrm{mg} / \mathrm{h}$, donnée expérimentale) sur une distance de $1 \mathrm{~cm}$. On calcule $\triangle\left|\psi_{e}\right|$ d'après la relation

$$
q=K \frac{\Delta\left|\psi_{e}\right|}{L} \quad(L=1 \mathrm{~cm})
$$

Estimation of soil water potential gradients near the seed required for maximum seed-soil water transfers

$$
\text { (1) after CAMPBELL (1974) }
$$

(2) soil water potential difference required for maximum seed-soil water transfers (distance $=1 \mathrm{~cm}) . \Delta\left|\psi_{e}\right|$ is calculated from the relation

\begin{tabular}{|c|c|c|c|c|c|c|c|c|c|}
\hline $\begin{array}{l}\text { Humidité initiale } \\
\text { du sol }\left(\mathrm{H}_{\mathrm{e}}\right)\end{array}$ & 0.06 & 0.08 & 0.10 & 0.12 & 0.14 & 0.16 & 0.18 & 0.20 & 0.22 \\
\hline $\begin{array}{l}\text { Potentiel hydrique } \\
\text { initial du sol } \\
\left(\left|\psi_{\mathrm{e}}\right| \text {, en bars }\right)\end{array}$ & 52 & 19.6 & 8.4 & 4.5 & 2.7 & 1.7 & 1.13 & 0.78 & 0.56 \\
\hline $\begin{array}{l}\text { Conductivité hydrique } \\
\text { du sol }(K, \text { en } \mathrm{m} / \mathrm{s})\end{array}$ & $1,1.10^{-13}$ & $1,4.10^{-12}$ & $9,7.10^{-12}$ & $4,8.10^{-11}$ & $1,9.10^{-10}$ & $6,2.10^{-10}$ & $1,7.10^{-9}$ & $4,4 \cdot 10^{-9}$ & $1,0.10^{-8}$ \\
\hline$\Delta$ ou $\left|\psi_{c}\right|$ (en bars) & 2360 & 187 & 26.1 & 5.2 & 1.3 & 0.42 & 0.15 & 0.06 & $\epsilon$ \\
\hline $\begin{array}{l}\text { Humidité finale } \\
\text { du sol après une heure } \\
\text { d'imbibition }\end{array}$ & 0.019 & 0.040 & 0.080 & 0.096 & 0.124 & 0.149 & 0.174 & 0.196 & 0.220 \\
\hline
\end{tabular}

$$
q=K \frac{\Delta\left|\psi_{e}\right|}{L} \quad(L=1 \mathrm{~cm})
$$

de celles obtenues lorsque $\mathrm{H}_{\mathrm{e}}=0,11\left(\left|\psi_{\mathrm{e}}\right| \sim 6,1\right.$ bars $)$ ou lorsque $\mathrm{H}_{\mathrm{e}}=0,083$ (palier hydrique sans germination).

La $2^{e}$ approche amène à situer à environ 3 bars la borne supérieure du domaine des potentiels $\left[0 ;\left|\psi_{\mathrm{e}}\right|\right]$ non limitant vis-à-vis de l'imbibition dans le cas du sol utilisé. En outre, la comparaison des 2 approches permet de valider a posteriori les critères arbitraires retenus lors du calcul d'ordre de grandeur. 


\section{CONDITIONS EXPÉRIMENTALES}

\section{A. Traitements expérimentaux et dispositifs}

Les traitements expérimentaux choisis visent à analyser le rôle des 2 groupes de variables de la relation (1) :

- d'une part, on cherche à placer les semences dans des conditions de potentiel hydrique variables. Pour cela, on réalise 3 profondeurs de semis $\left(Z_{1}=2,5 \mathrm{~cm} ; Z_{2}=\right.$ $4,5 \mathrm{~cm} ; \mathrm{Z}_{3}=6,5 \mathrm{~cm}$ ) dans un sol soumis au dessèchement ou préalablement irrigué : le traitement correspondant à la profondeur $Z_{2}$ est ainsi réalisé selon 2 variantes, l'une sèche $\left(Z_{2}\right)$, l'autre irriguée $\left(Z_{2 \mathrm{i}}\right)$.

On essaie de réaliser un état structural fin et homogène (état $\mathrm{S}_{0}$ ) et les lits de semences sont protégés des précipitations naturelles grâce à des tunnels en plastique.

- d'autre part, on cherche à placer les semences dans des conditions où l'état structural à leur contact est variable, tant par la porosité structurale que par la granulométrie des fragments terreux (" contact terre-graine » a priori variable). On réalise ainsi 3 états structuraux contrastés $\left(S_{1}, S_{2}, S_{3}\right.$, du plus fin au plus grossier) en localisant les semences à une profondeur où les conditions d'alimentation hydrique sont a priori non limitantes (profondeur $\mathrm{Z}_{2}$, cas 1 du tableau 1).

Enfin, chaque traitement réalisé est subdivisé en 2 zones, l'une étant tassée, l'autre non. Les traitements $S_{0}$ d'une part, $S_{1}, S_{2}, S_{3}$ d'autre part, ayant été réalisés à 2 dates différentes, ont subi des conditions climatiques différentes. La nomenclature des traitements est donnée par le tableau 3.

\section{B. Principaux contrôles réalisés}

L'unité expérimentale de base est une placette d'environ $20 \mathrm{~cm}$ de long, 4 à $5 \mathrm{~cm}$ de large et contenant au moins 3 semences. Chaque traitement est réalisé sur 2 séquences réparties au hasard dans 2 blocs et chaque séquence contient 20 placettes dont les dates d'observation et de prélèvement ont été randomisées.

1. Caractérisation de l'état structural (STENGEL, 1979).

La détermination de la porosité structurale $\left(\mathrm{n}_{\mathrm{s}}\right)$ est obtenue à partir des mesures de densité in situ par gammamétrie (SOANE et al., 1971) et des mesures de densité texturale sur agrégats (MONNIER et al., 1973).

Chaque traitement est caractérisé par une valeur moyenne, obtenue à partir de plusieurs répétitions, compte tenu de l'impossibilité de mesurer la porosité structurale à proximité de chaque semence (contraintes liées au temps et à la géométrie de la mesure par gammamétrie).

On constate (tabl. 4) que la gamme des porosités structurales réalisée dans tous les traitements est de faible amplitude $\left(\overline{\mathbf{n}}_{\mathrm{s}}=0,31, \sigma=0,03, \mathrm{n}=12\right)$, probablement à cause de l'ordre de grandeur limité des pressions appliquées lors du roulage $\left(300\right.$ à $\left.600 \mathrm{~g} / \mathrm{cm}^{2}\right)$. Les observations visuelles montrent que l'état strucural $\mathrm{S}_{\mathrm{o}}$ est généralement fin et homogène, conformément à l'objectif visé ; cependant, il existe une concentration des fragments grossiers en surface, triés lors de la préparation des lits de semences : dans le cas du traitement $Z_{1} S_{o}$, les semences se trouvent alors placées dans cette couche.

TABLEAU 3

Nomenclature des traitements expérimentaux

Design of experiments

(l) $i=$ irrigation

\begin{tabular}{|c|c|c|c|c|c|c|}
\hline Profondeur de semis & $2,5 \mathrm{~cm}$ & \multicolumn{4}{|c|}{$4,5 \mathrm{~cm}$} & $6,5 \mathrm{~cm}$ \\
\hline Etat structural & $\mathrm{S}_{\mathrm{o}}$ & $\mathrm{S}_{\mathrm{o}}$ & $S_{1}$ & $\mathrm{~S}_{2}$ & $\mathrm{~S}_{3}$ & $\mathrm{~S}_{\mathrm{o}}$ \\
\hline Nom du traitement & $\mathrm{Z}_{1} \mathrm{~S}_{\mathrm{o}}$ & $\begin{array}{c}\mathrm{Z}_{2} \mathrm{~S}_{\mathrm{o}} \\
\mathrm{Z}_{2 \mathrm{j}} \mathrm{S}_{\mathrm{o}}(1)\end{array}$ & $Z_{2} S_{1}$ & $\mathrm{Z}_{2} \mathrm{~S}_{2}$ & $\mathrm{Z}_{2} \mathrm{~S}_{3}$ & $\mathrm{Z}_{3} \mathrm{~S}_{\mathrm{o}}$ \\
\hline
\end{tabular}

Tassé + non tassé (pour chaque traitement)

TABLEAU 4

Etats structuraux des lits de semences (entre parentheses, nombre de mesures). Seeds-beds structure (within brackets, number of measurements).

\begin{tabular}{|c|c|c|c|c|c|c|c|}
\hline TRAITEMENTS & $\mathrm{Z}_{1} \mathrm{~S}_{\mathrm{o}}$ & $\mathrm{Z}_{2} \mathrm{~S}_{\mathrm{o}}$ & $\mathrm{Z}_{2 \mathrm{i}} \mathrm{S}_{\mathrm{o}}$ & $\mathrm{Z}_{2} \mathrm{~S}_{1}$ & $\mathrm{Z}_{2} \mathrm{~S}_{2}$ & $\mathrm{Z}_{2} \mathrm{~S}_{3}$ & $\mathrm{Z}_{3} \mathrm{~S}_{\mathrm{o}}^{(\mathrm{a})}$ \\
\hline $\begin{array}{l}\text { Porosité structurale moyenne } \mathrm{n}_{\mathrm{s}} \\
\quad \text { (traitements tassés) }\end{array}$ & $\begin{array}{c}0,31 \\
(7)\end{array}$ & $\begin{array}{c}0,31 \\
(7)\end{array}$ & $\begin{array}{c}0,29 \\
(1)\end{array}$ & $\begin{array}{c}0,29 \\
(5)\end{array}$ & $\begin{array}{c}0,29 \\
(5)\end{array}$ & $\begin{array}{c}0,28 \\
(5)\end{array}$ & $\begin{array}{c}0,17 \\
(7)\end{array}$ \\
\hline $\begin{array}{l}\text { Porosité structurale moyenne } \mathrm{n}_{\mathrm{s}} \\
\text { (traitements non tassés) }\end{array}$ & $\begin{array}{c}0,38 \\
(7)\end{array}$ & $\begin{array}{c}0,32 \\
(7)\end{array}$ & $\begin{array}{c}0,35 \\
(2)\end{array}$ & $\begin{array}{c}0,30 \\
(5)\end{array}$ & $\begin{array}{c}0,29 \\
(5)\end{array}$ & $\begin{array}{c}0,35 \\
(5)\end{array}$ & $\begin{array}{c}0,23 \\
(7)\end{array}$ \\
\hline $\begin{array}{l}\text { Proportion de terre fine } \\
\text { (volume \%) }\end{array}$ & $5-10$ & $65-70$ & $65-70$ & 80 & $40-50$ & $20-30$ & $65-70$ \\
\hline Taille modale des mottes (mm) & $8-10$ & $4-8$ & $4-8$ & $2-4$ & $3-6$ & $5-10$ & $4-8$ \\
\hline Répartition de la taille des mottes & hétérogène & homogène & homogène & homogène & $\begin{array}{l}\text { très hétérogène } \\
\text { (grosses mottes } \\
\text { en surface) }\end{array}$ & homogène & homogène \\
\hline
\end{tabular}


Dans le cas des états structuraux $S_{1}, S_{2}, S_{3}$, le passage du semoir aboutit à placer les semences en contact avec une certaine quantité de terre fine, atténuant ainsi le contraste entre traitements.

L'utilisation de (2) qui ne prend en compte que l'estimateur $n_{s}$ entraînera des estimations de $S_{1} / S_{t}$ proches pour des distributions dimensionnelles d'agrégats variables. Cette utilisation amènera ainsi à remettre éventuellement en cause cette relation $S_{1} / S_{t}=f\left(1-n_{s}\right)$ pour certaines tailles modales d'éléments structuraux.

\section{Potentiels hydriques}

Pour les semences, on détermine la teneur en eau massique de chacune d'elles après prélèvement et on utilise la relation $\left|\psi_{\mathrm{s}}\right|=\mathrm{f}\left(\mathrm{H}_{\mathrm{s}}\right)$ précédemment établie (BRUCKLER, 1983 I).

$$
\left|\psi_{\mathrm{s}}\right|=1098_{\mathrm{e}}^{-} 18,5 \mathrm{H}_{\mathrm{s}} \quad\left(\mathrm{r}^{2}=0,994\right)
$$

En ce qui concerne le sol, on mesure la teneur en eau massique d'une couche d'environ $1 \mathrm{~cm}$ d'épaisseur centrée sur la profondeur moyenne des semences d'une placette et de 2 à $3 \mathrm{~cm}$ de large; on se réfère ensuite à une relation $\left|\psi_{\mathrm{e}}\right|=\mathrm{f}\left(\mathrm{H}_{\mathrm{e}}\right)$ déterminée expérimentalement (presse à membrane et mise en équilibre avec une phase gazeuse, en dessication) :

Pour $\left|\psi_{\mathrm{e}}\right| \leqslant 16$ bars, $\left|\psi_{\mathrm{e}}\right|=0,0032 \mathrm{H}_{\mathrm{e}}-3,420\left(\mathrm{r}^{2}=\right.$ $0,981)$

Pour $\left|\psi_{\mathrm{e}}\right|>16$ bars, $\left|\psi_{\mathrm{e}}\right|=0,0035 \mathrm{H}_{\mathrm{e}}-3,412\left(\mathrm{r}^{2}=\right.$ $0,977)$

Les valeurs moyennes des teneurs en eau et du potentiel de l'eau du sol pour les différents traitements figurent au tableau 5 (on n'a représenté que les traitements tassés, les traitements non tassés étant très proches de ces derniers) : les semis superficiels sont très desséchés $\left(\left|\psi_{\mathrm{e}}\right|>100\right.$ bars $)$ tandis que les semis plus profonds correspondant à l'état $\mathrm{S}_{\mathrm{o}}(4,5 \mathrm{~cm} ; 6,5 \mathrm{~cm})$ sont situés sans ambiguïté dans le domaine non limitant $\left(\left|\psi_{\mathrm{e}}\right| \ll 3\right.$ bars $)$. Les traitements $S_{1}, S_{2}, S_{3}$ présentent des potentiels un peu plus élevés en valeur absolue que ces derniers, parfois situés à proximité de la limite séparant les domaines limitant et non limitant. Dans tous les cas, l'hétérogénéité des teneurs en eau à l'intérieur d'un traitement peut masquer l'évolution chronologique des profils hydriques d'un jour à l'autre.

\section{Contrôle de la température.}

La température est mesurée à l'aide de thermocouples cuivre-constantan placés à la profondeur moyenne de semis. Les figures 2 et 3 indiquent de fortes amplitudes thermiques, notamment pendant les premières 24 heures (les minimas nocturnes résultent de mesures effectuées à 0 et $-10 \mathrm{~cm}$ de la surface dans une station située à quelques kilomètres du lieu expérimental). On sera donc amené à adapter le modèle prévisionnel d'imbibition (relation (1) en envisageant l'influence de la température sur les différents termes de la relation (1).

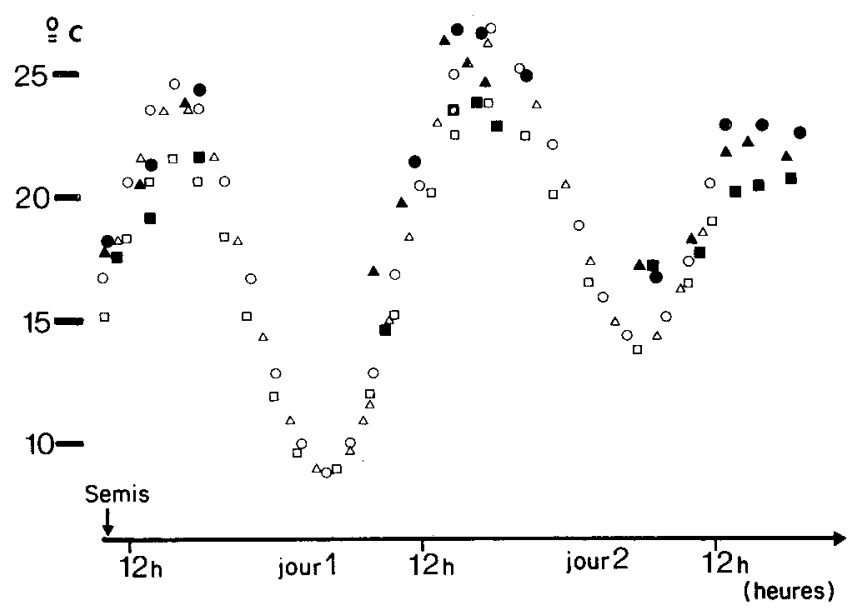

Figure 2

Variations de température dans les lits de semences (état structural $\left.S_{\alpha}\right)$ : Variations of the temperature in the seeds-beds (structure $S_{o}$ ):

$\begin{array}{ccc}z & \text { Mesures } & \text { Valeurs ajustées } \\ 2,5 \mathrm{~cm} & (\bullet) & (O) \\ 4,5 \mathrm{~cm} & (\Delta) & (\Delta) \\ 6,5 \mathrm{~cm} & (\square) & (\square)\end{array}$

TABLEAU 5

Teneurs en eau $\left(H_{e}\right)$ et potentiels hydriques $\left|\psi_{e}\right|$ à la profondeur moyenne des semences. Soil-water contents $\left(H_{e}\right)$ and soil-water potentials $\left(\left|\psi_{e}\right|\right)$ in the vicinity of the seeds.

\begin{tabular}{|c|c|c|c|c|c|c|}
\hline \multirow{3}{*}{ Traitement } & \multicolumn{3}{|c|}{$1^{\text {er }}$ jour (1) } & \multicolumn{3}{|c|}{$2^{e}$ jour $(2)$} \\
\hline & \multicolumn{2}{|c|}{$\begin{array}{c}\text { Teneur en eau } \\
\left(\mathrm{H}_{\mathrm{e}}\right)\end{array}$} & \multirow{2}{*}{$\begin{array}{l}\begin{array}{c}\text { Potentiel en bars } \\
\left(\left|\psi_{\mathrm{e}}\right|\right)\end{array} \\
{ }^{\mu} \\
211\end{array}$} & \multicolumn{2}{|c|}{$\begin{array}{l}\text { Teneur en eau } \\
\qquad\left(\mathbf{H}_{e}\right)\end{array}$} & \multirow{2}{*}{$\begin{array}{c}\begin{array}{c}\text { Potentiel en bars } \\
\left(\left|\psi_{\mathrm{e}}\right|\right)\end{array} \\
\stackrel{\mu}{177}\end{array}$} \\
\hline & $\begin{array}{c}\mu \\
0,039\end{array}$ & $\stackrel{\sigma}{0,010}$ & & $\stackrel{\mu}{0,041}$ & $\begin{array}{c}\sigma \\
0,011\end{array}$ & \\
\hline $\mathrm{Z}_{2} \mathrm{~S}_{\mathrm{o}}$ & 0,226 & 0,010 & 0,5 & 0,219 & 0,009 & 0,6 \\
\hline $\mathrm{Z}_{2 \mathrm{i}} \mathrm{S}_{\mathrm{o}}$ & 0,218 & 0,006 & 0,6 & 0,212 & 0,008 & 0,6 \\
\hline $\mathrm{Z}_{2} \mathrm{~S}_{1}$ & 0,179 & 0,015 & 1,1 & 0,181 & 0,019 & 1,0 \\
\hline $\mathrm{Z}_{2} \mathrm{~S}_{2}$ & 0,164 & 0,021 & 1,4 & 0,155 & 0,017 & 1,8 \\
\hline $\mathrm{Z}_{2} \mathrm{~S}_{3}$ & 0,164 & $0,0 ! 7$ & 1,4 & 0,159 & 0,019 & 1,7 \\
\hline $\mathrm{Z}_{3} \mathrm{~S}_{\mathrm{o}}$ & 0,241 & 0,006 & 0,4 & 0,232 & 0,011 & 0,5 \\
\hline
\end{tabular}




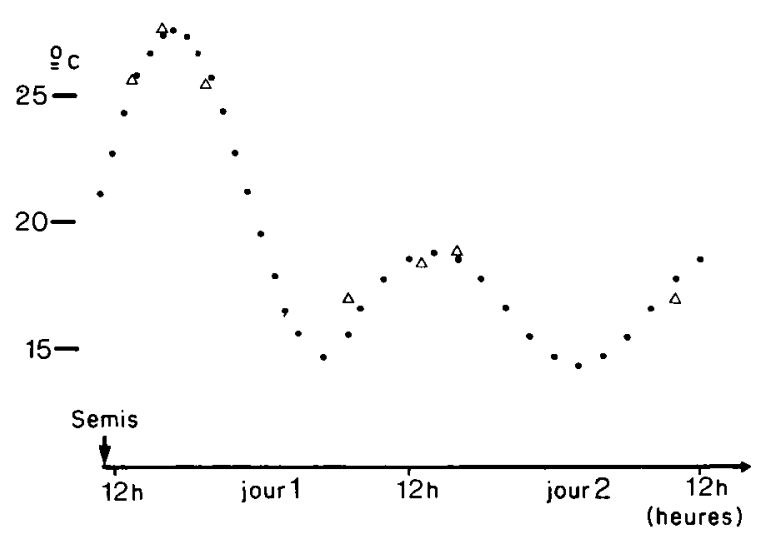

Figure 3

Variations de température dans les lits de semences (états structuraux $\left.S_{1}, S_{2}, S_{3}\right)$.

Variations of temperature in the seeds-beds (structures $S_{1}, S_{2}, S_{3}$ ).

$(\Delta$ mesures, measurements

$(\bullet)$ valeurs ajustées, fitted values

De plus, pour disposer d'une fonction continue $T=f(t)$ pendant toute la durée de l'imbibition, les mesures ont été ajustées à des fonctions du type $\mathrm{T}=\mathrm{a}+\mathrm{b} \sin \left(2 \pi \frac{\mathrm{t}}{\mathrm{p}}\right)$, quels que soient l'expérience, la profondeur de semis ou le jour considérés ( $p=$ période de $24 \mathrm{~h}$, $t$ en heures). Ces fonctions seront utilisées lors de la modélisation de l'imbibition des semences et sont représentées sur les figures 2 et 3 .

\section{RÉSULTATS ET DISCUSSION}

\section{A. Rôle du potentiel hydrique sur l'imbibition et la germination}

\section{Recherche d'un estimateur de l'environnement hydrique} de la semence.

La localisation des semences en profondeur présente une certaine hétérogénéité, alors qu'elles sont précisément placées dans une zone présentant de très forts gradients hydriques (fig. 4 et 5 ). Il en résulte qu'une petite variation de position peut induire de très grandes variations du potentiel de l'eau au contact des semences et que la prévision de l'imbibition et de la germination, conformément au tableau 1 , ne peut se faire à partir de valeurs moyennes de potentiel hydrique $\left|\psi_{\mathrm{e}}\right|$ (tabl. 5).

On est ainsi amené à estimer, pour chaque semence, la valeur du potentiel de la zone où elle est localisée. Pour cela, on repère la position de chaque semence par rapport à des gradients de coloration induits par des gradients de teneur en eau du sol.

On a pu distinguer, dans un profil hydrique en voie de dessèchement, 3 zones superposées (de haut en bas) :

- une zone de couleur claire (value 6 du code MUNSELL)

- une zone de couleur intermédiaire, bien délimitée dans sa partie supérieure et plus diffuse vers le bas. L'épaisseur de cette couche est relativement constante $(10 \mathrm{~mm})$.

- une zone de couleur sombre (value $3 \mathrm{du}$ code MUNSELL).

Il s'avère (fig. 6) que ces différentes zones correspondent à des domaines bien distincts de teneur en eau : la couche claire correspond ainsi à des humidités $\mathrm{H}_{\mathrm{e}}$ comprises entre 0,02 et $0,07\left(2000>\left|\psi_{\mathrm{e}}\right|>50\right.$ bars $)$, la couche intermédiaire à des humidités variant de 0,07 à $0,14\left(50>\left|\psi_{\mathrm{e}}\right|>\right.$
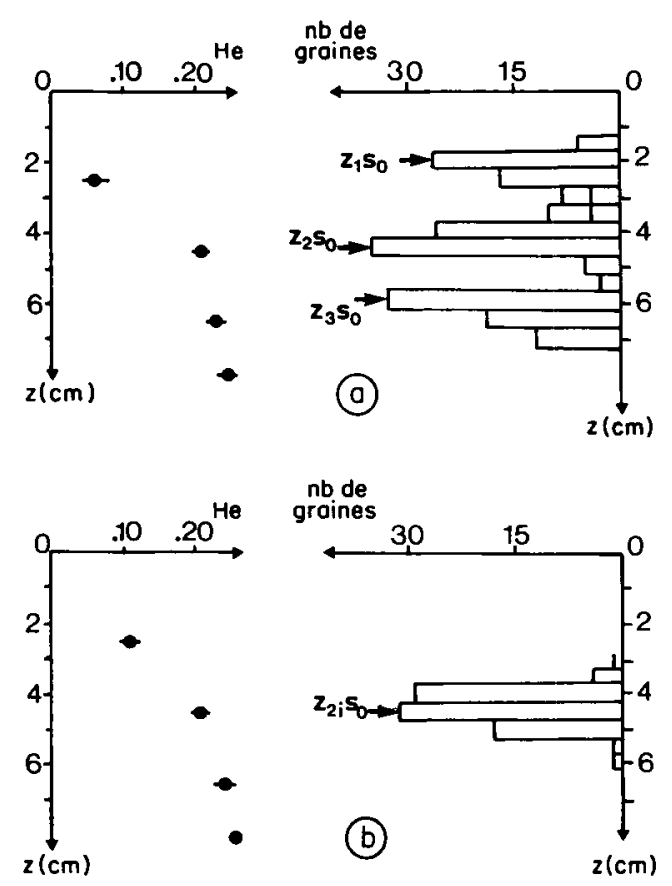

Figure 4

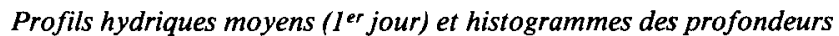
de semis : Etat structural $S_{o}$; a sans et $b$ avec irrigation (les fleches indiquent les classes modales de profondeurs de semis pour chaque traitement, tassés et non tassés réunis))

Mean soil-water contents (first day) and histogram of seed's depths : Structure $S_{o}$ a without and $b$ with irrigation (arrows show where is the most important percentage of seeds for each treatment, grouping compacted or not).

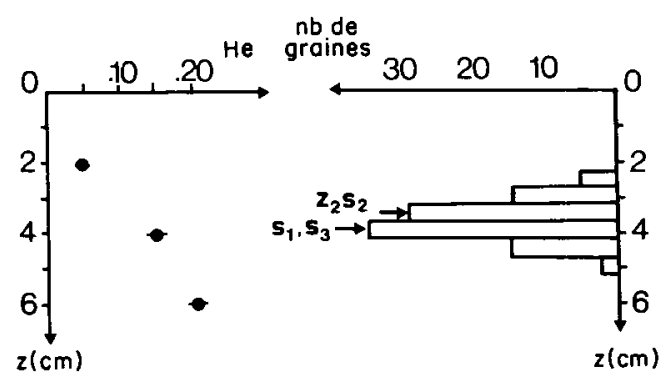

Figure 5

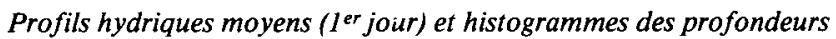
de semis : Etats structuraux $S_{1}, S_{2}, S_{3}$. (Les flèches indiquent les classes modales de profondeurs de semis pour chaque traitement).

Mean soil-water contents (first day) and histogram of seed's depths : Structures $S_{1}, S_{2}, S_{3}$.

(Arrows show where is the most important percentage of seeds for each treatmont, compacted or not).

3 bars), la couche sombre correspondant à des valeurs $\left|\psi_{\mathrm{e}}\right|$ toujours inférieures à 3 bars (domaine non limitant).

En situant chaque semence dans l'une des 3 zones, on peut alors connaître la gamme des potentiels $\left|\psi_{\mathrm{e}}\right|$ existant à son contact, pour les états structuraux $S_{1}, S_{2}, S_{3}$, on a précisé la notation et noté systématiquement la distance $c$ de la semence au front de dessèchement qui est la limite nette entre les 2 premières couches.

\section{Prévisions et résultats expérimentaux.}

\section{a) Imbibition.}

- le cas 1 (domaine non limitant, tabl. 1) doit être réalisé pour les semences situées en zone sombre $\left(\left|\psi_{\mathrm{e}}\right|<3\right.$ bars) : les semences sont celles des semis les plus profonds 


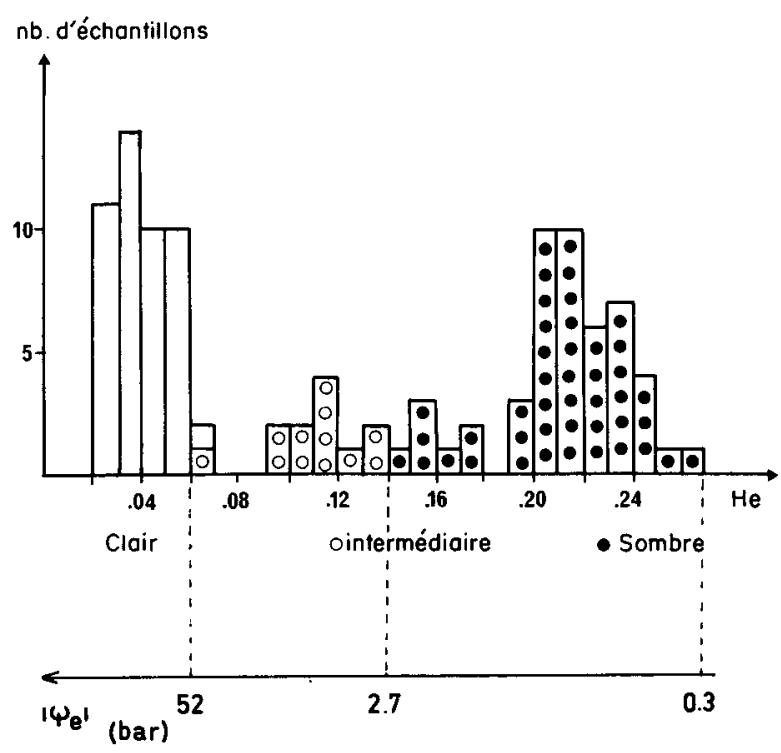

Figure 6

Coloration du lit de semences et humidité pondérale.

Colour of seed's bed and soil water-content.

ou préalablement irrigués $\left(Z_{2 i} S_{o}, Z_{3} S_{o}\right)$, en partie celles du traitement $\mathrm{Z}_{2} \mathrm{~S}_{\mathrm{o}}$; on peut adjoindre au cas 1 les semences du traitement $Z_{2} S_{1}$, si la cote à laquelle elles sont placées est située à plus de $15 \mathrm{~mm}$ en dessous du front de dessèchement.

On constate (tabl. 6) que les semences des traitements cités ont, au moment des observations, déjà atteint ou dépassé l'humidité critique de la semence en dessous de laquelle la germination est impossible $\left(\mathrm{H}_{\mathrm{c}}=0,25\right.$ à 0,26$):$ l'hypothèse d'appartenance de ces semences au cas 1 est donc parfaitement plausible, d'ailleurs corroborée par l'homogénéité de leur teneur en eau et par les cinétiques expérimentales obtenues pour une température moyenne de $20^{\circ} \mathrm{C}$ en domaine non limitant (fig. 1).

- le cas $2\left(3<\left|\psi_{\mathrm{e}}\right|<16\right.$ bars, tabl. 1), doit nécessairement se rencontrer dans la couche intermédiaire, pour des semences suffisamment éloignées du front de dessèchement, mais à moins de $15 \mathrm{~mm}$ en dessoús de celui-ci. On peut prévoir qu'appartiennent à ce cas certaines semences du traitement $\mathrm{Z}_{2} \mathrm{~S}_{\mathrm{o}}$ (zone intermédiaire) et du traitement $\mathrm{Z}_{2} \mathrm{~S}_{1}$ si
$C \leqslant 15 \mathrm{~mm}$. La situation observée ne contredit pas la prévision, puisqu'on observe simultanément des cas 1 et 2 pour le traitement $\mathrm{Z}_{2} \mathrm{~S}_{\mathrm{o}}$ (couche sombre ou intermédiaire), des cas 2 et 3 pour $Z_{2} S_{1}$ (couche intermédiaire).

- le cas 3 (16 bars $<\left|\psi_{\mathrm{e}}\right|$, tabl. 1) doit être réalisé pour toutes les semences placées en zone claire et pour une partie de celles situées en zone intermédiaire, à proximité du front de dessèchement. L'observation montre sans ambiguïté que toutes les semences des traitements correspondant aux semis superficiels $\left(Z_{1} S_{0}\right.$, tassé et non tassé) sont situés en zone claire. Pour ces semences, les humidités atteintes au moment de l'observation sont très inférieures à $H_{c}$ et très hétérogènes (tabl. 6). Des prélèvements postérieurs à ceux mentionnés dans le tableau 6 amènent aux mêmes conclusions : à $\mathrm{t}=220 \mathrm{~h}, \mathrm{H}_{\mathrm{S}}=0,148, \sigma \mathrm{H}_{\mathrm{s}}=0,035$. $\mathrm{La}$ prévision n'est donc pas contredite et la dispersion des valeurs $\left|\psi_{\mathrm{s}}\right|$ s'accorde bien avec la gamme de potentiels hydriques $\left|\psi_{\mathrm{e}}\right|$ dans la zone où sont placées les semences : ainsi, pour un domaine $\left|\psi_{\mathrm{s}}\right|$ de [27 bars ; 640 bars], on trouve un domaine de potentiels $\left|\psi_{\mathrm{e}}\right|$ correspondant de [60 bars ; 610 bars]. On peut donc considérer qu'il y a recouvrement entre ces 2 gammes de variation et l'hypothèse selon laquelle les semences de ces traitements atteignent un palier hydrique sans germination peut être conservée.

\section{b) Germination.}

En fin d'expérience, le prélèvement des semences restantes permet d'estimer les taux de germination et les stades atteints (tabl. 7 et 8). Les prévisions issues du tableau 1 sont nettement confirmées pour les traitements situés en zone claire et en zone sombre ; même au bout de $220 \mathrm{~h}$, les semences situées en zone claire ne germent pas. La variabilité observée dans le traitement $\mathrm{Z}_{2} \mathrm{~S}_{\mathrm{o}}$ (couche sombre ou intermédiaire, tabl. 7) et pour les semences de la couche intermédiaire des états $S_{1}$ à $S_{3}$ (tabl. 8), n'est pas surprenante compte tenu de l'importance que revêt dans ces cas la position exacte de la semence dans le profil : c'est ainsi que l'on peut observer des taux variables de semences non germées dans la couche intermédiaire correspondant à de légères variations de positionnement entre les échantillons prélevés (tabl. 8).

Au total, la confrontation entre prévision et observations, portant sur des critères essentiels du point de vue agronomique (possibilité ou non de germination, durée moyenne

\section{TABLEAU 6}

Teneurs en eau des semences avant apparition des premières germinations.

(1) $c$ : distance de la semence au front de dessèchement.

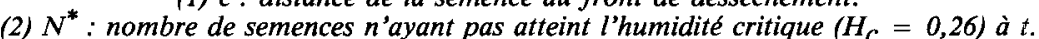
$n$ : nombre de semences observées.

Seeds water-contents before the first germination.

(1) $c$ : distance between seeds and drying front.

(2) $N^{*}$ : number of seeds with water-contents below the critical level $\left(H_{c}=0,26\right)$ at $t$. $n$ : number of observed seeds.

\begin{tabular}{|c|c|c|c|c|c|c|c|c|c|c|c|}
\hline \multirow{2}{*}{ Traitements } & & \multicolumn{2}{|c|}{$\mathrm{Z}_{1} \mathrm{~S}_{\mathrm{o}}$} & \multicolumn{2}{|c|}{$\mathrm{Z}_{2} \mathrm{~S}_{\mathrm{o}}$} & \multicolumn{2}{|c|}{$\mathrm{z}_{2 \mathrm{i}} \mathrm{S}_{\mathrm{o}}$} & \multicolumn{2}{|c|}{$\mathrm{Z}_{2} \mathrm{~S}_{1}$} & \multicolumn{2}{|c|}{$\mathrm{Z}_{3} \mathrm{~S}_{\mathrm{o}}$} \\
\hline & & tassé & non tassé & tassé & non tassé & tassé & non tassé & $\underset{c \leqslant 15 \mathrm{~mm}}{\text { tassé }}+$ & $\begin{array}{l}\text { non tassé) } \\
\qquad>15 \mathrm{~mm}^{(1)}\end{array}$ & tassé & non tassé \\
\hline Durée d'imbibition (t) & & & & $29 \mathrm{~h} \leqslant$ & $t \leqslant 31 \mathrm{~h}$ & & & $21 \mathrm{~h} \leqslant$ & $\leqslant t \leqslant 26 h$ & $29 \mathrm{~h} \leqslant$ & $\leqslant 31 \mathrm{~h}$ \\
\hline \multirow{2}{*}{$\begin{array}{l}\text { Teneur en eau } \\
\text { des semences } \\
\text { à } t\end{array}$} & $\mu$ & 0,058 & 0,073 & 0,271 & 0,248 & 0,273 & 0,276 & 0,237 & 0,278 & 0,285 & 0,263 \\
\hline & $\sigma$ & 0,075 & 0,058 & 0,024 & 0,027 & 0,012 & 0,020 & 0,023 & 0,015 & 0,017 & 0,010 \\
\hline$N^{*} / \mathrm{n}$ & (2) & $11 / 11$ & $11 / 11$ & $3 / 14$ & $6 / 9$ & $2 / 13$ & $2 / 14$ & $17 / 20$ & $1 / 17$ & $0 / 11$ & $3 / 13$ \\
\hline
\end{tabular}


TABLEAU 7

Stades des semences après $80 \mathrm{~h}$ de séjour en terre : état structural $S_{O}$. Stages of the seeds after $80 \mathrm{~h}$ in seeds-beds : seeds-bed structure $S_{O}$.

\begin{tabular}{|c|c|c|c|c|}
\hline \multirow{2}{*}{$\begin{array}{c}\text { Traitements } \\
\text { expérimentaux }\end{array}$} & \multirow{2}{*}{$\begin{array}{c}\text { Nombre } \\
\text { de semences } \\
\text { observées }\end{array}$} & \multicolumn{3}{|c|}{$\begin{array}{l}\text { Stades observés } \\
\text { (en } \% \text { de l'effectif total) }\end{array}$} \\
\hline & & Non germées & $\begin{array}{l}\text { Radicule } \\
\text { seule } \\
\text { apparente }\end{array}$ & $\begin{array}{c}\text { Radicule et } \\
\text { tigelle } \\
\text { apparentes }\end{array}$ \\
\hline $\begin{array}{l}Z_{1} S_{0} \\
\text { (couche claire) }\end{array}$ & 39 & 98 & 2 & 0 \\
\hline $\mathrm{Z}_{2} \mathrm{~S}_{\mathrm{o}}$ & 59 & 14 & 30 & 56 \\
\hline $\begin{array}{l}\mathrm{Z}_{2 \mathrm{i}} \mathrm{S}_{\mathrm{o}}, \mathrm{Z}_{3} \mathrm{~S}_{\mathrm{o}} \\
\text { (couche sombre) }\end{array}$ & 58 & 0 & 5 & 95 \\
\hline
\end{tabular}

TABLEAU 8

Stades des semences après 55 et $80 \mathrm{~h}$ de séjour en terre : états structuraux $S_{1}, S_{2}, S_{3}$.

Stages of the seeds after $55 \mathrm{~h}$ and $80 \mathrm{~h}$ in seeds-beds : seeds-beds structures $S_{1}, S_{2}, S_{3}$

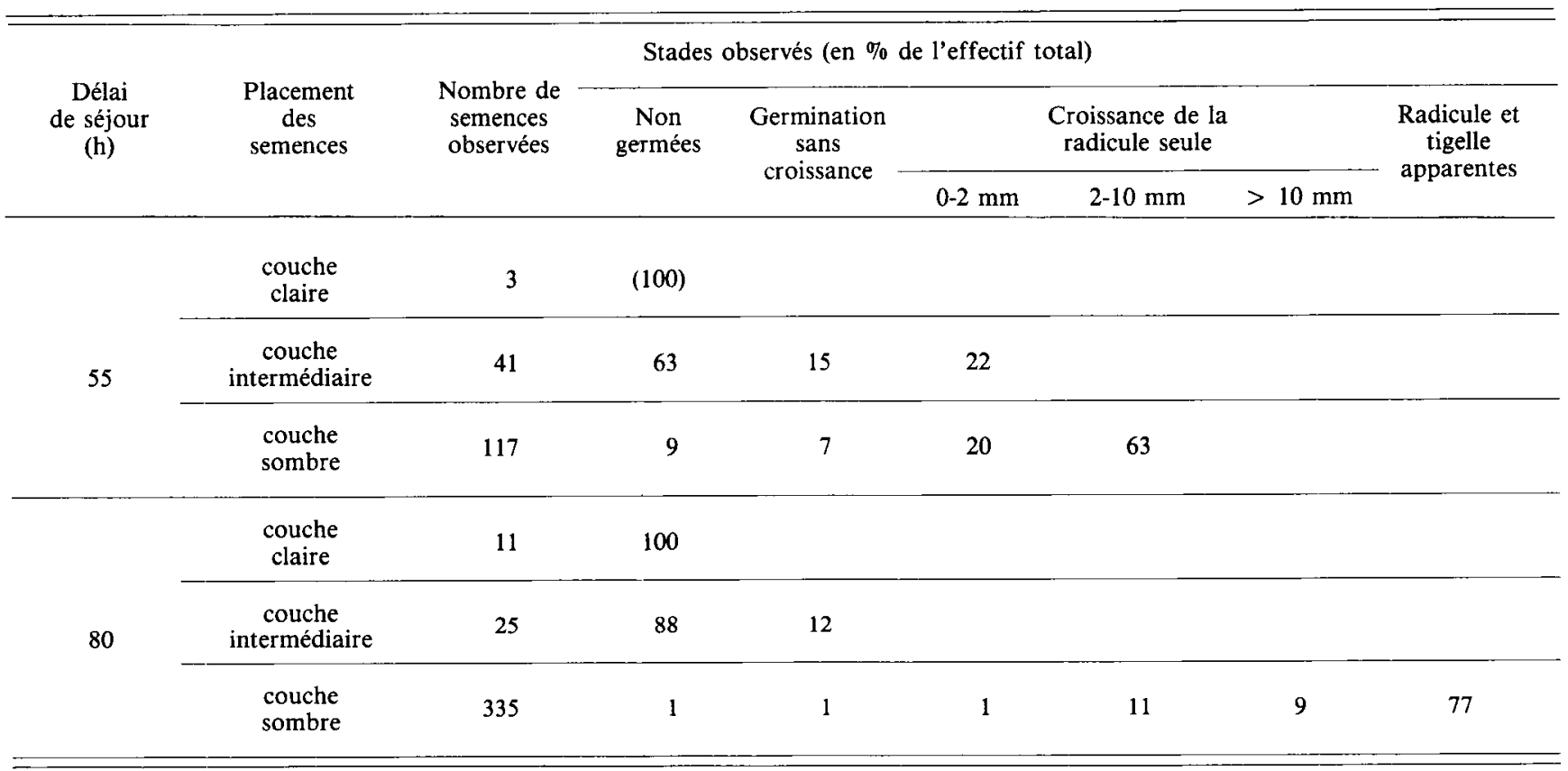

d'humectation jusqu'à $H_{c}$ ) est présentée par le tableau 9 (la prévision est effectuée à partir de la relation (1) adaptée à des conditions non isothermes - cf. plus loin). Du point de vue d'éventuelles applications, le degré d'accord obtenu est très satisfaisant.

\section{B. Rôle de l'état structural sur la cinétique d'imbibition. Prévisions et observations}

La construction de cinétiques prévisionnelles d'imbibition pour différents états structuraux et leur confrontation aux résultats expérimentaux suppose la réalisation de 3 conditions :

1. Ne considérer que des semences situées dans le domaine non limitant (tabl. 1), afin que seule la surface humectée en phase liquide ou vapeur détermine la cinétique observée.
2. Adapter au préalable le modèle (relation (1)) à la prise en compte de variations de températures.

3. Estimer pour les cas expérimentaux choisis, une valeur moyenne $\left(S_{1}, S_{v}\right)$, et un intervalle de confiance pour un risque donné.

1. Prise en compte des variations de température dans la modélisation

En se référant à la relation (1), on çonstate que les termes sensibles aux variations de température sont les suivants :

$$
K^{\prime}\left(H_{s}\right), D^{\prime}\left(H_{s}\right),\left(\left|\psi_{s}\right|-\left|\psi_{e}\right|\right),\left(p_{s}-p_{e}\right)
$$

a) Cas des termes $K^{\prime}\left(H_{s}\right)$ et $D^{\prime}\left(H_{s}\right)$

Ces 2 fonctions dépendent de l'humidité de la semence et de la température : $s i$ on considère 2 températures $T_{1}$ et 
TABLEAU 9

Prévisions et observations des comportements des semences.

$$
\text { (a) } S_{o} \text {; (b) } S_{1}, S_{2}, S_{3} \text {. }
$$

(1) d'après la relation (1) en conditions non isothermes.

(2) d'après ajustement statistique sur les points expérimentaux.

Predicted and observed responses of seeds. (a) $S_{0}$; (b) $S_{1}, S_{2}, S_{3}$

(l) from the relation (I) for non-isothermal conditions. (2) from statistical fiting of experimental data.

\begin{tabular}{|c|c|c|c|c|c|}
\hline $\begin{array}{c}\text { Domaine de teneur } \\
\text { en eau }\end{array}$ & Non limitant & \multicolumn{4}{|c|}{ Limitant } \\
\hline $\begin{array}{l}\text { Domaine des potentiels }\left|\psi_{\mathrm{e}}\right| \\
\text { correspondants }\end{array}$ & $\left|\psi_{\mathrm{e}}\right|<3$ bars (cas 1$)$ & \multicolumn{4}{|c|}{$\left|\psi_{\mathrm{e}}\right|>16$ bars (cas 3$)$} \\
\hline $\begin{array}{l}\text { Cas } \\
\text { expérimentaux } \\
\text { correspondants }\end{array}$ & $\begin{array}{cc}(\mathrm{a}) & (\mathrm{b}) \\
\mathrm{Z}_{2 \mathrm{i}} \mathrm{S}_{\mathrm{o}}, \mathrm{Z}_{3} \mathrm{~S}_{\mathrm{o}}: & \text { zone sombre : } \\
\text { toutes semences } & \text { toutes semences } \\
\mathrm{Z}_{2} \mathrm{~S}_{\mathrm{o}}: & \\
\text { quelques } & \\
\text { semences } & \end{array}$ & $\begin{array}{c}\text { (a) } \\
\mathrm{Z}_{2} \mathrm{~S}_{\circ}: \\
\text { quelques } \\
\text { semences }\end{array}$ & $\begin{array}{c}\text { (b) } \\
\text { zone } \\
\text { intermédiaire : } \\
\text { certaines } \\
\text { semences }\end{array}$ & $\begin{array}{c}\text { (a) } \\
\mathrm{Z}_{1} \mathrm{~S}_{\mathrm{o}} \\
\text { toutes semences }\end{array}$ & $\begin{array}{c}\text { (b) } \\
\text { zone claire: } \\
\text { toutes semences } \\
\text { zone } \\
\text { intermédiaire : } \\
\text { certaines } \\
\text { semences }\end{array}$ \\
\hline $\begin{array}{l}\text { Potentiels expérimentaux }\left|\psi_{\mathrm{e}}\right| \\
\text { moyens }\end{array}$ & $\left|\psi_{\mathrm{e}}\right|<0.7$ bar $\left|1 \leqslant \psi_{\mathrm{e}}\right|<2.2$ bars & $3<\left|\psi_{\mathrm{e}}\right|<16$ bars & $3<\left|\psi_{\mathrm{e}}\right|<16$ bars & $\left|\psi_{\mathrm{e}}\right|>100$ bars & $\left|\psi_{\mathrm{e}}\right|>100$ bars \\
\hline \multirow{2}{*}{$\begin{array}{r}\text { GERMINATION } \frac{\text { prévue : }}{\text { observée : }} \\
\text { (taux de germination } \\
\text { à } 80 \mathrm{~h} \text { ) }\end{array}$} & Possible & Possible & Possible & Impossible & Impossible \\
\hline & $\begin{array}{cc}\mathrm{Z}_{2 \mathrm{i}} \mathrm{S}_{\mathrm{o}}, \mathrm{Z}_{3} \mathrm{~S}_{\mathrm{o}} & \text { couche sombre } \\
100 \% & 99 \%\end{array}$ & $\begin{array}{l}\mathrm{Z}_{2} \mathrm{~S}_{\mathrm{o}}: \\
\text { germination } \\
\text { hétérogène }\end{array}$ & $\begin{array}{c}\text { zone } \\
\text { intermédiaire : } \\
\text { germination } \\
\text { hétérogène }\end{array}$ & $\begin{array}{l}\mathrm{Z}_{1} \mathrm{~S}_{\mathrm{o}}: \\
2 \%\end{array}$ & $\begin{array}{c}\text { zone claire : } \\
0 \% \\
\text { zone } \\
\text { intermédiaire : } \\
\text { existence de } \\
\text { semences non } \\
\text { germées }\end{array}$ \\
\hline \multirow{2}{*}{ IMBIBITION $\begin{array}{r}\frac{\mathrm{H}_{\mathrm{s}}=\mathrm{H}_{\mathrm{c}}^{(1)}}{} \\
\text { observée : } \\
\text { (heure où } \\
\left.\mathrm{H}_{\mathrm{s}}=\mathrm{H}_{\mathrm{c}}\right)_{(2)}\end{array}$} & $25 \mathrm{~h}$ & - & - & $\begin{array}{c}\text { Palier } \\
\mathrm{H}_{\mathrm{s}}<\mathrm{H}_{\mathrm{c}}\end{array}$ & $\begin{array}{c}\text { Palier } \\
\mathrm{H}_{\mathrm{s}}<\mathrm{H}_{\mathrm{c}}\end{array}$ \\
\hline & $28 \mathrm{~h}$ & - & - & $\begin{array}{l}\mathrm{H}_{\mathrm{s}}<\mathrm{H}_{\mathrm{c}} \\
(\mathrm{à} 220 \mathrm{~h})\end{array}$ & $\begin{array}{l}\mathrm{H}_{\mathrm{s}}<\mathrm{H}_{\mathrm{c}} \\
(\text { à } 80 \mathrm{~h})\end{array}$ \\
\hline
\end{tabular}

$\mathrm{T}_{2}$, on obtient les relations suivantes (BRUCKLER, 1979):

$$
\begin{gathered}
\mathrm{K}^{\prime}\left(\mathrm{H}_{\mathrm{s}}, \mathrm{T}_{2}\right)=\frac{\mu_{1}}{\mu_{2}} . \mathrm{K}^{\prime}\left(\mathrm{H}_{\mathrm{s}}, \mathrm{T}_{1}\right) \\
\mu=\text { viscosité de l'eau } \\
\text { et D' }\left(\mathrm{H}_{\mathrm{s}}, \mathrm{T}_{2}\right)=\left(\frac{\mathrm{T}_{2}}{\mathrm{~T}_{1}}\right) 1,75 . \mathrm{D}^{\prime}\left(\mathrm{H}_{\mathrm{s}}, \mathrm{T}_{1}\right)
\end{gathered}
$$

b) Cas des termes relatifs au sol $\left(\left|\psi_{\mathrm{e}}\right|, \mathrm{p}_{\mathrm{e}}\right)$

En milieu non limitant, on peut toujours faire l'approximation $\left|\psi_{\mathrm{e}}\right| \sim 0$ compte tenu de l'ordre de grandeur du terme $\left|\psi_{\mathrm{s}}\right|$. Par contre, dans les mêmes conditions, $\mathrm{p}_{\mathrm{e}}$ est toujours très voisin de $p_{0}$, pression de vapeur saturante de l'eau à la température $\mathrm{T}$. On écrira donc :

$$
\mathrm{p}_{\mathrm{e}}=\mathrm{p}_{\mathrm{e}}(\mathrm{T}) \sim \mathrm{p}_{0}(\mathrm{~T})=2,26.10-5 \mathrm{e}^{-0,0063 \mathrm{~T}}
$$

$\left(r^{2}=0,999\right), 280 \mathrm{~K}<\mathrm{T}<300 \mathrm{~K}$ (d'après les données $\mathrm{du}$ « Handbook of Physics and Chemistry »).

c) Cas des termes relatifs à la semence $\left(\psi_{\mathrm{s}}, \mathrm{p}_{\mathrm{s}}\right)$

Nous admettrons, au vu des rares références expérimentales disponibles (Collis-GeORGE \& MELville, 1978), l'hypothèse d'indépendance des termes $\psi_{\mathrm{s}}, \mathrm{p}_{\mathrm{s}}$ vis-à-vis de la température.
Enfin, c'est à partir des relations $T=f(t)$ (fig. 2,3 ) et des relations (3), (4), (5) qu'on pourra prendre en compte a priori, pas à pas, l'effet de la température sur la cinétique d'imbibition $\mathrm{H}_{\mathrm{s}}=\mathrm{f}(\mathrm{t})$.

\section{Estimation des surfaces d'imbibition $\left(S_{l}, S_{v}\right)$}

Le principe d'estimation de $S_{1}$ revient à utiliser la relation (2), en se plaçant nécessairement dars un domaine non limitant et ceci bien que les états structuraux considérés ne soient pas identiques à ceux ayant présidé à l'établissement de (2).

$\mathrm{Si}$, en $1^{\text {re }}$ approche; on néglige l'effet des traitements, les valeurs moyennes de la porosité structurale $n_{s}$ et les intervalles de confiance des valeurs individuelles sont les suivants :

- état structural $S_{0}: n_{s 1}=31$ p. $100,[23$ p. 100 ; 40 p. 100]

(à 95 p. 100)

- états structuraux $\mathrm{S}_{1}, \mathrm{~S}_{2} \mathrm{~S}_{3}: \mathrm{n}_{\mathrm{s} 2}=30$ p. $100,[22$ p. $100 ; 38$ p. 100$]$ (à 95 p. 100).

Par ailleurs, la relation (2), $\mathrm{S}_{1} / \mathrm{S}_{\mathrm{t}}=\mathrm{f}\left(\mathrm{n}_{\mathrm{s}}\right)$, comporte ellemême un certain degré d'incertitude : à la borne supérieure 
de l'intervalle de confiance des valeurs expérimentales $n_{s}$ (borne inférieure pour $\left(1-n_{s}\right)$ ) correspond un intervalle de confiance des valeurs individuelles $S_{1} S_{t}$ (fig. 7); soit $S_{1} S_{t}$ INF sa borne inférieure. De même, considérant la borne inférieure de l'intervalle de confiance de $n_{s}$ borne supérieure pour $\left(1-n_{s}\right)$ ), on définira $S_{\curlywedge} / S_{t}$ sup. On obtient ainsi un intervalle $\left[S_{\mathrm{I}} / \mathrm{S}_{\mathrm{tINF}} ; \mathrm{S}_{\mathrm{l}} / \mathrm{S}_{\mathrm{tsup}}\right]$ à l'extérieur duquel une valeur $S_{l} / S_{t}$ a une très faible probabilité d'existence : même pour des valeurs de porosité peu probables (cas des abscisses a et $b$ de la figure 7), il existe des valeurs $S_{S} / S_{t}$ dont la probabilité d'appartenance au domaine défini est encore élevé. Ces domaines sont les suivants :

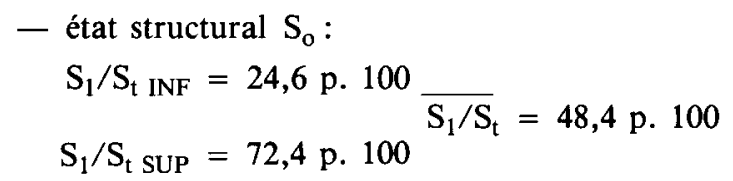

- états structuraux $\mathrm{S}_{1}, \mathrm{~S}_{2}, \mathrm{~S}_{3}$ :

$$
\begin{aligned}
& \mathrm{S}_{1} / \mathrm{S}_{\mathrm{t} \text { INF }}=25,8 \text { p. } 100 \\
& \mathrm{~S}_{1} / \mathrm{S}_{\mathrm{t} \mathrm{SUP}}=73,4 \text { p. } 100 \\
& \overline{S_{1} / S_{t}}=49,5 \text { p. } 100
\end{aligned}
$$

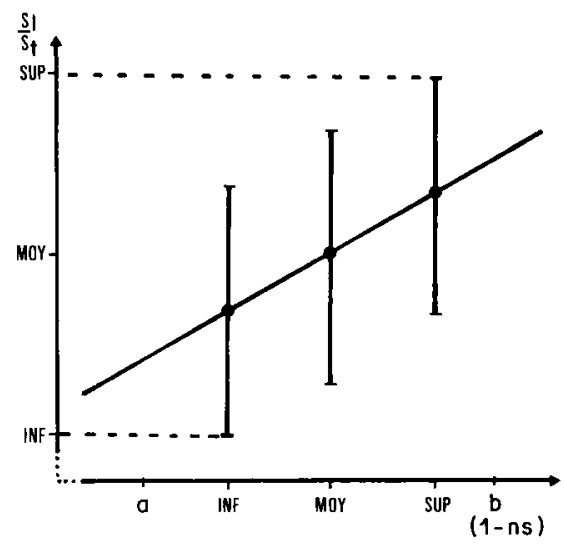

Figure 7

Estimation d'un intervalle $\left[S_{I} / S_{t I N F} ; S_{I} / S_{t} S U P\right]$ à partir de la relation $S_{1} / S_{t}=f\left(I-n_{s}\right)$.

Estimation of an interval $\left[S_{1} / S_{t I N F} ; S_{1} / S_{t} S_{U P}\right.$ from the relation $S_{l} / S_{t}=f\left(I-n_{S}\right)$.

\section{Confrontation de la localisation des points expérimentaux avec les cinétiques prévisionnelles}

Par un calcul pas à pas $(\Delta t=0,25 \mathrm{~h})$ qui permet de prendre en compte l'effet des variations de T sur l'imbibition (cf. IV - B.1.), on construit 3 cinétiques pour chaque expérience correspondant respectivement aux valeurs $S_{1} / S_{t}$ INF $\mathrm{S}_{1} / \mathrm{S}_{\mathrm{t} \text { MOY }}, \mathrm{S}_{1} / \mathrm{S}_{\mathrm{t}}$ SuP (fig. 8 et 9 ).

Globalement, la répartition des points expérimentaux (semences toujours situées en zone sombre) est compatible avec l'allure des cinétiques prévues : on entend par là que la teneur en eau moyenne des semences, à un moment donné, est proche de celle correspondant à la cinétique prévisionnelle moyenne, et que les amplitudes des variations des teneurs en eau des semences sont de même ordre de grandeur que l'amplitude correspondant au faisceau prévisionnel. Cependant, respectivement 14 et 31 p. 100 des points expérimentaux pour les traitements $S_{0}$ ou $S_{1}, S_{2}, S_{3}$ se situent à l'extérieur de ces faisceaux, en majorité en dessus de ceux-ci.

Il y a donc divergence entre prévisions et observations, dont on peut chercher les différentes origines :

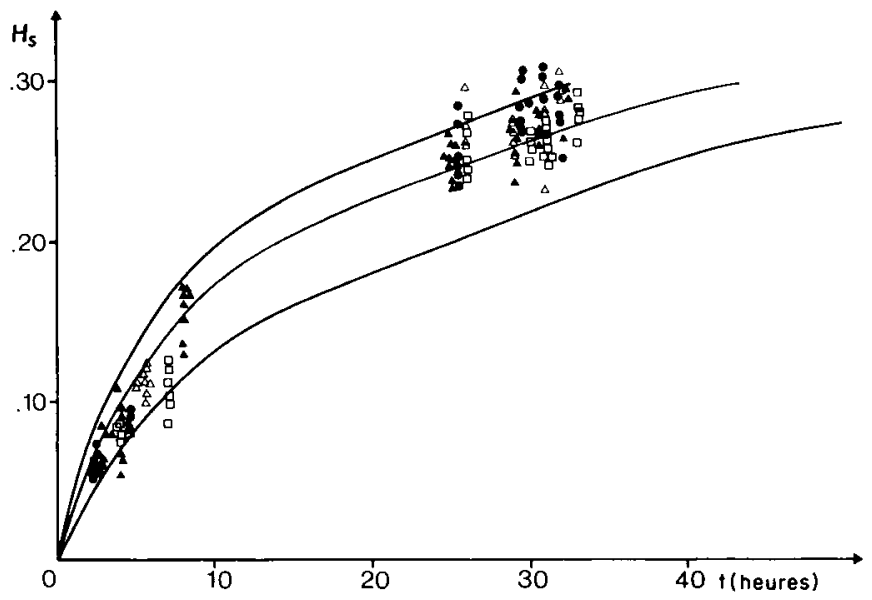

Figure 8

Cinétiques d'imbibition prévisionnelles et points expérimentaux :

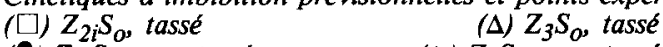

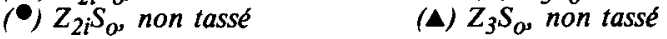

Predicted values of imbibition and experimental data :

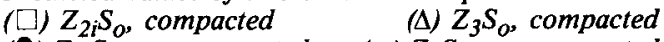

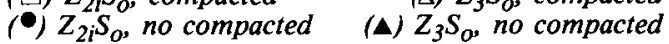

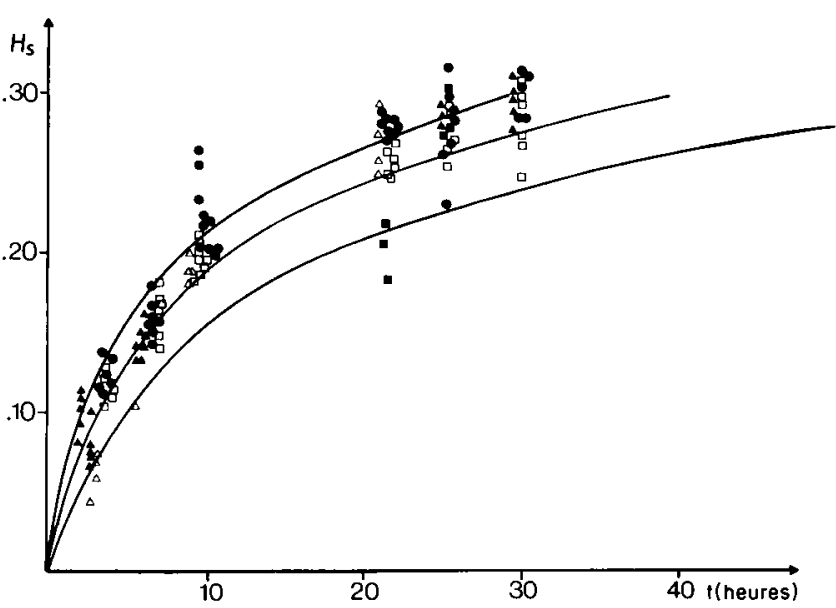

Figure 9

Cinétiques d'imbibition prévisionnelles et points expérimentaux :

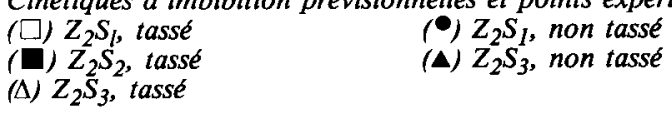

Predicted values of imbibition and experimental data :

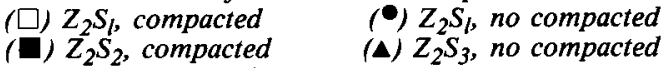

(A) $Z_{2} S_{3}$, compacted

a) Erreurs dues à une mauvaise estimation $\mathrm{S}_{1} / \mathrm{S}_{t}$

L'estimation $S_{1} / S_{t}$ est l'objet d'une double incertitude : d'une part, elle se fonde sur un modèle physique du « contact terre-graine » établi pour des volumes homogènes d'agrégats de petite taille ( 2 à $3 \mathrm{~mm}$ ), ce qui $a$ priori, doit conditionner la validité de la relation (2). D'autre part, l'estimation de $n_{s}$ peut être entachée d'erreur (erreur liée à la discordance entre le volume de mesure et le volume déterminant $S_{1}$ au contact de la semence par exemple). L'examen des figures 8 et 9 suggère que ces 2 sources d'erreurs ne jouent pas un rôle majeur : en effet, les modifications de cinétiques dues à une variation des termes $S_{1} / S_{t}$ (toutes choses égales par ailleurs) sont d'une allure différente des divergences constatées et excluent notamment un recoupement des cinétiques. 
b) Erreurs dues à une mauvaise estimation du potentiel des semences $\left(\left|\psi_{\mathrm{s}}\right|=\mathrm{f}\left(\mathrm{H}_{\mathrm{s}}\right)\right)$

Ces erreurs seraient alors liées à l'influence de la modification du lot de semences par rapport à celui ayant permis d'établir la relation $\left|\psi_{\mathrm{s}}\right|=\mathrm{f}\left(\mathrm{H}_{\mathrm{s}}\right)$ et/ou à l'hétérogénéité possible des potentiels initiaux des semences.

c) Erreurs dues à une mauvaise prise en compte des variations de température

Pendant les 10 premières heures de l'imbibition, on constate que l'écart entre les 2 cinétiques prévisionnelles moyennes est nettement plus faible qu'entre les 2 cinétiques réelles moyennes. Or, ce sont les conditions de température du lit de semences qui distinguent principalement les 2 expériences pendant cette période : environ 3 à $5^{\circ} \mathrm{C}$ de plus pour la deuxième.

Cette constatation suggère qu'une partie importante de la divergence entre prévisions et observations provient d'une prise en compte incomplète des fluctuations de température sur les cinétiquès prévisionnelles.

Les erreurs liées à l'ajustement $\mathrm{T}=\mathrm{f}(\mathrm{t})$ sont peu probables ou, du moins, de faible amplitude, puisque les conséquences d'une variation de température de 2 à $3^{\circ} \mathrm{C}$ (ordre de grandeur des erreurs d'ajustements) sur une cinétique prévisionnelle sont d'un ordre de grandeur nettement inférieur aux divergences observées.

C'est plutôt sur le plan théorique que l'influence des variations de température sur les termes du modèle est probablement mal cernée : en effet, seules les modifications liées à l'état physique de l'eau (viscosité, pression de vapeur saturante par exemple) ont été intégrées. On est amené à remettre en cause l'hypothèse selon laquelle les fluctuations de température n'entraînent pas de modifications physiologiques sensibles des semences vis-à-vis de leur comportement d'absorbants par l'intermédiaire de fonctions $\left|\psi_{s}\right|\left(\mathrm{H}_{s}, T\right), \varrho_{s}\left(\mathrm{H}_{s}, T\right), K^{\prime}\left(\mathrm{H}_{s}, T\right)$, D' $\left(H_{s}, T\right)$.

\section{CONCLUSION}

Le principal objet du travail entrepris était le contrôle au champ de la valeur prédictive d'un modèle d'imbibition des semences de maïs (BRUCKLER, 1983, II). A cet égard, 3 points essentiels se dégagent :
1. Les réponses des semences aux variations de potentiels hydriques du sol, qui se traduisent par des cas-types d'imbibition et de germination, ont été correctement prévues.

2. La relation (2) n'a pu être testée sur une large gamme de porosités structurales, mais pour des lits de semences où la répartition des calibres d'éléments structuraux était variable. Pour cette faible gamme de porosités structurales, la relation (2) semble rendre compte de façon satisfaisante de l'influence de l'état structural du lit de semences sur l'imbibition.

3. Les variations de température semblent introduire une distorsion sensible entre les prévisions et les résultats expérimentaux, qui pourrait être due à la non prise en compte des effets physiologiques de ces variations. Une amélioration de la valeur prévisionnelle du modèle nécessiterait alors une meilleure connaissance de l'influence de la température sur les propriétés d'absorption des semences.

Vis-à-vis de l'imbibition, le placement des semences est de loin le facteur de variation le plus discriminant ; au champ, le repérage des gradients de coloration s'est révélé, en texture limoneuse, un bon indicateur des gradients hydriques et des valeurs de potentiels correspondantes.

L'état structural intervient avant tout sur la profondeur et la régularité du semis et, probablement, sur le régime de dessication obtenu en conditions climatiques déterminées. On rejoint ici les observations de FENNECH \& PAPY (1977), COZIC \& SebillotTE (1976). Par contre, les variations de « contact terre-graine ", souvent privilégiées, ne sont pas nettement apparues : d'une part, les tassements ont été réalisés sur terre sèche avec des pressions modérées (situation fréquente dans la pratique), d'autre part, l'élément semeur homogénéise la répartition des calibres d'éléments structuraux au contact immédiat de la semence.

Une dernière application de l'ensemble des travaux présentés consisterait à prévoir, à partir des données climatiques, les risques de mauvaise germination. Dans cette perspective, une prévision fine des profils hydriques dans différents lits de semence et sous des conditions climatiques connues serait nécessaire.

Reçu le 29 décembre 1981. Accepté le 22 novembre 1982.

\section{RÉFÉRENCES BIBLIOGRAPHIQUES}

Bruckler L., 1979. L'imbibition et la germination des semences en conditions sèches : Influence des caracteres physiques du lit de semences. Thèse de docteur-ingénieur I.N.A.-P.G., 119 p.

Bruckler L., 1983. Rôle des propriétés physiques du lit de semences sur l'imbibition et la germination: (I) Elaboration d'un modèle du « système terre-graine ". Agronomie, 3, 3.

Bruckler L., 1983. Rôle des propriétés physiques du lit de semences sur l'imbibition et la germination : (II) Contrôle expérimental d'un modèle d'imbibition des semences et possibilités d'applications. Agronomie, 3,3 .

Campbell Gaylon S., 1974. A simple method for determining unsaturated conductivity from moisture retention data. Soil Sci., 117, 6 .

Collis-George N., Melville M.D., 1978. Water absorption by swelling seeds. Surface condensation boundary conditions. Aust. J. Soil Res., 16, 291-310.

Coxic P., Sebillotte M., 1976. Etude au champ de la germination-levée du maïs en conditions sèches. Ronéo, Chaire d'Agronomie I.N.A.P.G., $18 \mathrm{p}$.
Dasberg S., 1971. Soil-water movement to germinating seeds. J. exp. Bot., 22, 73, 999-1008.

Fennech J., Papy F., 1977. Conditions de réussite de la levée en climat méditerranéen. Cas des cultures de céréales en sec au Nord du Maroc. Ann. agron., 28 (6), 599-635.

Monnier G., Stengel P., Fies J.-C., 1973. Une méthode de mesure de la densité apparente de petits agglomérats terreux. Application à l'analyse des systèmes de porosité du sol. Ann agron., 24 (5), 533-545. Soane B.D., Campbell D.J., Herkes S.M., 1971. Handheld gammaray transmission equipment for the measurement of bulk density of field soils. J. agric. Eng. Res., 16, 146-156.

Stengel P., 1979. Utilisation de l'analyse des systèmes de porosité pour la caractérisation de l'état physique du sol in situ. Ann. agron., 30 (1), $27-51$. 